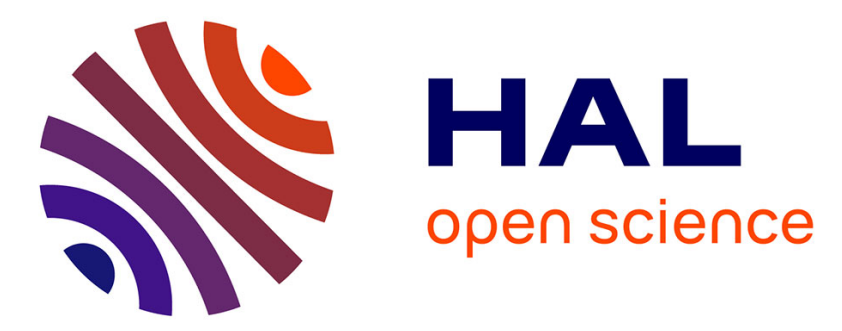

\title{
Modélisation multiplicative des perturbations dans un modèle de réapprovisionnement sur deux périodes
}

Selma-Afakh Khader, Yacine Rekik, Valerie Botta-Genoulaz, Jean-Pierre

Campagne

\section{- To cite this version:}

Selma-Afakh Khader, Yacine Rekik, Valerie Botta-Genoulaz, Jean-Pierre Campagne. Modélisation multiplicative des perturbations dans un modèle de réapprovisionnement sur deux périodes. Journal Européen des Systèmes Automatisés (JESA), 2013, 47 (4-8), pp.589-615. hal-01355971

\section{HAL Id: hal-01355971 \\ https://hal.science/hal-01355971}

Submitted on 2 Feb 2020

HAL is a multi-disciplinary open access archive for the deposit and dissemination of scientific research documents, whether they are published or not. The documents may come from teaching and research institutions in France or abroad, or from public or private research centers.
L'archive ouverte pluridisciplinaire HAL, est destinée au dépôt et à la diffusion de documents scientifiques de niveau recherche, publiés ou non, émanant des établissements d'enseignement et de recherche français ou étrangers, des laboratoires publics ou privés. 


\title{
Modélisation multiplicative des perturbations dans un modèle de réapprovisionnement sur deux périodes
}

\author{
Selma-Afakh KHADER ${ }^{1}$, Yacine REKIK ${ }^{2}$, Valérie BOTTA- \\ GENOULAZ $^{1}$, Jean-Pierre CAMPAGNE ${ }^{1}$
}

1. INSA Lyon, DISP

Bâtiment Léonard de Vinci, 21, Avenue Jean Capelle, 69621 Villeurbanne, France \{selma-afakh.khader, valerie.botta, jean-pierre.campagne\}@insa-lyon.fr

2. EM Lyon Business school, DISP

23, av. Guy de Collonges, 69134 Ecully, France

rekik@em-lyon.com

RESUME. La majorité des articles traitant de la problématique de gestion de stock partent de l'hypothèse d'une égalité parfaite entre la quantité de produits que montre le Système d'Information et la quantité réellement disponible. Cette hypothèse est infirmée par certaines études empiriques qui ont mis en évidence l'existence de perturbations qui peuvent entraîner un écart entre ces deux valeurs. Ces perturbations peuvent avoir un impact direct sur la satisfaction de la demande dans le contexte d'un e-détaillant. Dans cet article, nous proposons un modèle de réapprovisionnement sur deux périodes visant à optimiser le profit dans ce contexte. Nous étendons la littérature en considérant une modélisation multiplicative des perturbations. Nous répondons aussi à des interrogations managériales concernant la gestion des risques liés à la modélisation des perturbations de stocks entre les deux périodes.

ABSTRACT. Most inventory models studied in the scientific literature assume implicitly that the inventory position shown in the information system is equal to the actual physical stock used to satisfy the clients' demands. But empirical studies highlighted that errors and inventory perturbations may occur in the inventory system. Such errors influence directly the demand satisfaction in an e-retailer context. In this paper, we propose a replenishment model for two selling periods in order to optimize the e-retailer profit considering inventory inaccuracy, with a multiplicative modeling of inventory errors. We also deduce managerial insights for sharing the risks related to inaccuracies.

MOTS-CLES: gestion de stock, modèle de réapprovisionnement, récursion arrière, perturbations, modèle multiplicatif des erreurs, e-détaillant.

KEYWORDS: inventory management, replenishment model, backward recursion, multiplicative modeling of error, e-retailer.

$\mathrm{RS}-\mathrm{JESA}-\mathrm{n}^{\circ} \mathrm{x} / 201 \mathrm{x}, \mathrm{xx}-\mathrm{xx}$ 


\section{Introduction}

La majorité des modèles développés en gestion de stock suppose une égalité parfaite entre la quantité que le Système d'Information (IS) montre et celle qui est réellement disponible dans le Stock Physique $(\mathrm{PH})$. En effet, les processus d'approvisionnement auprès du fournisseur, de réception, de comptage et stockage des produits ainsi que la mise à jour du IS sont implicitement supposés parfaits et synchronisés et non sujets à des erreurs administratives, humaines ou informatiques.

Cependant plusieurs facteurs peuvent perturber la synchronisation implicitement supposée du IS et du PH et créer ainsi une différence qui, si non prise en compte, peut impacter directement la performance économique ainsi que la satisfaction des clients. Cette différence de quantité entre IS et PH, communément connue dans la littérature sous l'appellation "gestion de stock sujet à des perturbations sur les quantités informationnel physique - inventory inaccuracy », peut engendrer un impact majeur sur la performance d'une entreprise (Raman et al., 2001).

Les sources des erreurs peuvent être classées comme suit (Schrady, 1970; Atali et al., 2009) :

- perturbations permanentes sur le $\mathrm{PH}$ : des produits peuvent être endommagés, périmés ou volés par les clients / employés dans un magasin / entrepôt de stockage sans que ce changement soit détecté et mis à jour dans le IS ; dans ce cas, la quantité visible dans le IS est supérieure à la quantité réellement disponible dans le $\mathrm{PH}$. L'impact de ce type de perturbations a été récemment étudié par (Xu, 2012);

- perturbations temporaires sur le $\mathrm{PH}$ : des produits peuvent être mal rangés dans le magasin / entrepôt de stockage ou être dans le mauvais emplacement ou rayon. Ces produits sont bien comptabilisés dans le IS mais sont temporairement indisponibles à la vente à cause de la non-connaissance de leur emplacement réel. A titre d'exemple, Ton et Raman (2010) et Rekik et al. (2008) ont présenté des travaux empiriques et théoriques sur cette source de perturbations temporaires en gestion de stock. Ici également, la quantité visible dans le IS est supérieure à la quantité disponible dans le $\mathrm{PH}$;

- perturbations de transaction, d'enregistrement ou de mise à jour du IS : ces erreurs apparaissent généralement lors des mouvements des stocks (réception d'une commande, son inspection et son enregistrement dans le système informatique : par exemple saisir un 7 au lieu d'un 5 au moment de l'enregistrement de la marchandise reçue) et elles impactent le niveau de stock dans le système d'information (DeHoratius et Raman, 2008). Dans ce cas, la quantité visible dans le IS peut être supérieure ou inférieure à la quantité réellement disponible dans le PH.

Rekik et al. (2008) ajoutent à ces trois sources de perturbation une quatrième liée aux erreurs associées au système de réapprovisionnement. En effet, les fournisseurs peuvent livrer une quantité différente de la quantité commandée. En absence d'une inspection lors de la réception de la marchandise, cette différence entre commandé / livré peut se transformer en une différence entre PH / IS. 
La plupart des investigations théoriques dans ce domaine de recherche (Sandoh et Shimamoto, 2001 ; Fleisch et Tellkamp, 2005 et Xu et al., 2012) considère généralement une seule source de perturbations. Rares sont les auteurs qui combinent plusieurs sources. Parmi eux, on trouve Nachtmann et al. (2001) et Kök et Shang (2008).

Il est également à noter que la synchronisation des quantités IS et $\mathrm{PH}$ peut se faire de deux façons :

- un inventaire physique dont le but est de mettre à jour la quantité dans le IS. Dans le cas d'un inventaire parfait, non sujet à des erreurs, les quantités PH et IS sont coordonnées (Kök et Shang, 2008),

- le déploiement d'une technologie avancée d'identification des produits telle que la technologie RFID qui permet de mieux tracer les produits dans un entrepôt de stockage et de limiter voire supprimer les sources de perturbations mentionnées plus haut (Atali et al., 2009).

Les perturbations en gestion de stock décrites précédemment ont été principalement étudiées dans le contexte du détaillant ou de la grande distribution, où le client final est supposé être présent physiquement dans le magasin afin de confronter sa demande aux quantités disponibles et exposées sur les rayons (quantité dans le PH). Dans ce contexte, la quantité dans le IS ne joue pas un rôle majeur dans le processus de satisfaction de la demande du client final. A contrario, dans un contexte e-détaillant ou e-commerce, le client final passe sa commande derrière un écran ou par téléphone et sa demande est directement confrontée à la quantité disponible dans le IS. Il est à noter qu'en dehors du détaillant, ce mode d'acceptation des commandes, qui se base principalement sur la quantité IS, correspond à la majorité des acteurs de la chaîne logistique qui fonctionnent en B-to-B.

Dans le contexte du e-détaillant, le IS joue un premier rôle dans le processus de satisfaction de la demande puisque la confrontation de la demande du client final à la quantité dans le IS engendre un engagement de vente. Cet engagement doit normalement par la suite être honoré lors de la livraison au client si la quantité IS traduit bien la quantité PH. Dans le cas de perturbations, si le stock PH est inférieur au stock IS, cet engagement n'est pas respecté, ce qui engendre des pénalités additionnelles. Pour la fonction économique du e-détaillant, il est à noter que la nonsatisfaction d'un engagement de vente est plus pénalisante qu'une vente non acceptée.

Pour modéliser les perturbations sur les quantités physique et informationnel, deux configurations existent dans la littérature :

- la configuration additive : les quantités IS et PH sont modélisées comme étant la quantité commandée auprès des fournisseurs à laquelle s'additionne une variable aléatoire qui caractérise les perturbations sur les quantités IS et PH ;

- la configuration multiplicative : dans ce cas, les quantités IS et PH sont modélisées sous la forme de la quantité commandée auprès des fournisseurs 
multipliée par une variable aléatoire qui caractérise les perturbations sur les quantités IS et PH.

Les perturbations de transaction ou d'enregistrement peuvent être modélisées comme additives car ces erreurs sont indépendantes de la quantité commandée auprès du fournisseur contrairement aux erreurs ayant comme sources le vol ou la détérioration d'un produit qui peuvent être modélisées comme multiplicatives car l'ampleur et la variabilité de ces erreurs dépendent de la quantité commandée auprès du fournisseur.

La majorité des travaux sur la prise en compte des perturbations en gestion de stock concerne le contexte du détaillant avec une modélisation additive des erreurs (Kök et Shang, 2008 ; Ton et Raman, 2010). Les travaux considérant le contexte edétaillant sont beaucoup moins nombreux. Rekik (2011) considère le contexte edétaillant avec une configuration additive des perturbations via un modèle de gestion de stock mono-période. Sahin et Dallery (2009) considèrent la configuration multiplicative des erreurs uniquement sur le SI dans un contexte également monopériodique. Khader et al. (2012) étendent les résultats des contributions précédentes vers le cas multiplicatif avec à la fois des perturbations sur les quantités IS et PH dans un contexte mono-période.

Sur la base de cette dernière référence, notre article contribue à la résolution du problème dans le contexte de l'e-détaillant avec une modélisation multiplicative des erreurs en proposant un modèle de gestion de stock à deux périodes (l'e-détaillant commande pour les deux périodes avant le début de la première période de vente). Le but de cette démarche est double :

- analyser les propositions managériales concernant les quantités à commander pour les deux périodes ;

- étudier la différence entre la gestion d'un système sujet à des perturbations sur plusieurs périodes et une succession de gestion mono-périodique.

Afin d'illustrer le contexte e-détaillant du point de vue gestion de stock, nous considérons dans le modèle à deux périodes une politique de re-complètement périodique. La séquence d'évènements pour chaque période est décrite ci-dessous et est illustrée dans Figure 1.

(1) En supposant que le stock initial en début de période de vente $i$ soit $x_{i}$, l'edétaillant commande auprès de son fournisseur une quantité $Q_{i}$ pour remplir son stock jusqu'à un niveau $Y_{i}$, on rappelle que le e-détaillant commande pour les deux périodes avant le début de la première période de vente et la réception des commandes se fait par période.

(2) Après réception de la marchandise, pour chaque période $i$ l'e-détaillant met à jour son système d'information en inspectant et en stockant les produits livrés dans son entrepôt de stockage.

(3) A cause des perturbations, la quantité PH (respectivement IS) réellement disponible en stock (respectivement visible dans le IS), notée par $Y_{P H i}$ 
(respectivement $Y_{I S i}$ ), inclut une erreur modélisée de façon stochastique et multiplicative en fonction du niveau de re-complètement $Y_{i}: Y_{P H i}=\gamma_{P H i} . Y_{i}$ (respectivement $\left.Y_{I S i}=\gamma_{I S i} . Y_{i}\right) . \quad \gamma_{P H i}$ et $\gamma_{I S i}$ étant deux variables aléatoires normalement distribuées qui caractérisent les perturbations sur les quantités PH et IS.

(4) Durant la période $i$, l'e-détaillant cumule les commandes des clients finaux reçues par voie électronique ou téléphonique et les confronte à la quantité observée dans le IS. En comparant la demande cumulée $D i$ et la quantité IS, il peut accepter ou refuser des commandes et s'engage vis-à-vis de ses clients. La quantité correspondant aux commandes acceptées, notées par $K_{i}$, est le minimum entre la demande cumulée reçue et le stock IS : $K_{i}=\operatorname{Min}\left(Y_{I S i}, D i\right)$.

(5) A la fin de la période $i$, l'e-détaillant procède à la livraison groupée des commandes acceptées $K_{i}$. Si la quantité PH est suffisante pour satisfaire $K_{i}$, il pourra honorer tous ses engagements. Sinon, il ne livre que la quantité à sa disposition et subit une pénalité additionnelle de non-respect de ses engagements. La quantité livrée, notée par $\operatorname{Liv}_{i}$ est le minimum entre les commandes acceptées et le stock physique : $\operatorname{Liv} v_{i}=\operatorname{Min}\left(Y_{P H i}, K_{i}\right)$.

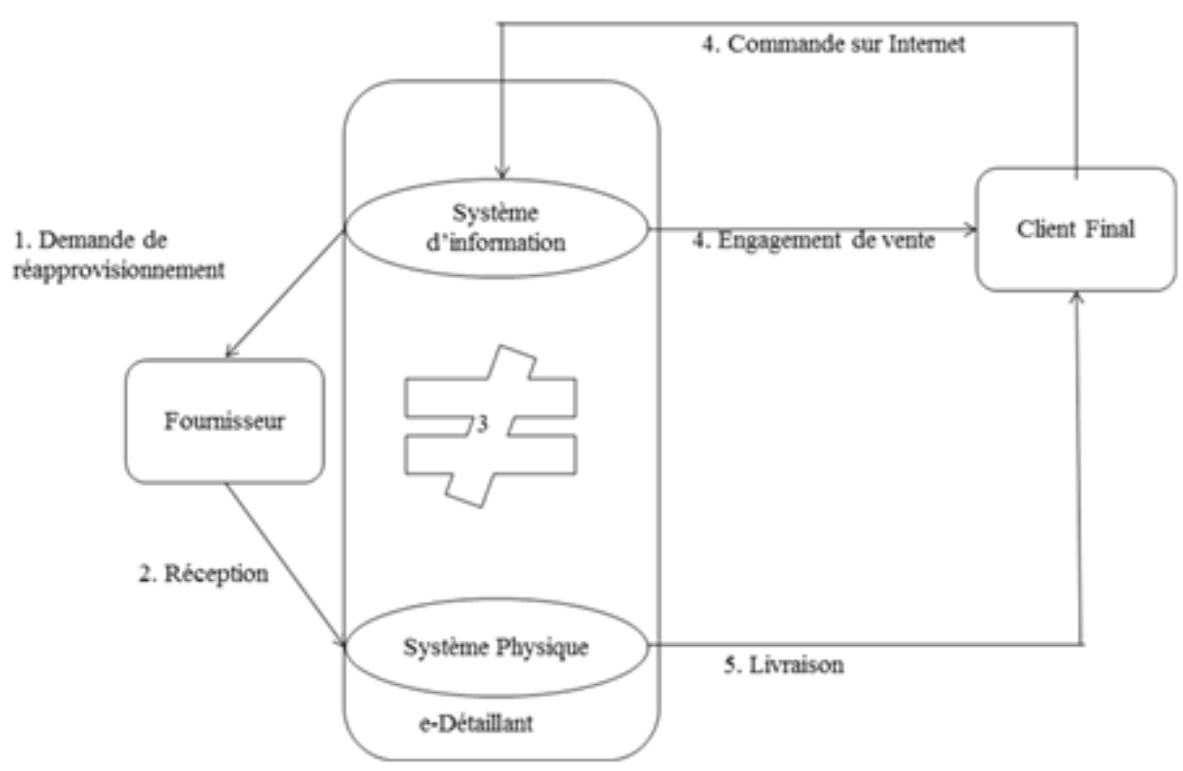

Figure.1. Système de gestion de stock d'un e-détaillant : séquence des événements 
La suite de cet article est organisée comme suit : dans la section 2 nous présentons l'approche utilisée pour modéliser le problème à deux périodes et pour optimiser la fonction profit moyen du e-détaillant. Dans la section 3 nous présentons les résultats d'une application numérique ainsi que les réponses à certaines interrogations managériales. Nous concluons l'article en Section 4 en proposant des pistes de recherche future.

\section{Modélisation et Optimisation}

Tout au long de cet article, nous utilisons les notations qui suivent :

Di :

$f_{D i}\left(\operatorname{resp} . F_{D i}\right):$

$\mu_{D i}:$

$\sigma_{D i}:$

$\gamma_{I S i}\left(\operatorname{resp} \cdot \gamma_{P H i}\right)$ :

$\sigma_{I S i}\left(\operatorname{resp} . \sigma_{P H i}\right)$ :

$f_{I S i}\left(\operatorname{resp} . F_{I S i}\right)$ :

$f_{P H i}\left(\operatorname{resp} . F_{P H i}\right):$
$\mu_{I S i}\left(\operatorname{resp} \cdot \mu_{P H i}\right):$

variable aléatoire représentant la demande durant la période $i=1,2$

fonction densité (resp. fonction de répartition) de la variable aléatoire $D i$ durant la période $i=1,2$

moyenne de $D i$ durant la période $i=1,2$

écart-type de la variable aléatoire $D i$ durant la période $i=1,2$

variable aléatoire représentant les perturbations sur la quantité IS (resp. PH) durant la période $i=1,2$

moyenne de $\gamma_{I S i}\left(\operatorname{resp} \cdot \gamma_{P H i}\right)$ durant la période $i=1,2$

écart type de $\gamma_{I S i}\left(\operatorname{resp} \cdot \gamma_{P H i}\right)$ durant la période $i=1,2$

fonction densité (resp. fonction de répartition) de $\gamma_{I S i}$ durant la période $i=1,2$

fonction densité (resp. fonction de répartition) de $\gamma_{P H i}$ durant la période $i=1,2$

$Y_{i}: \quad$ niveau de re-complètement pour la période $i=1,2$

$Y_{i}^{*}: \quad$ valeur optimale $Y_{i}$ de la période $i=1,2$

$Y_{I S i}: \quad$ quantité affichée dans le système d'information au début de la période $i=1,2$

$Y_{P H i}: \quad$ quantité disponible dans le système physique au début de la période $i=1,2$

$x_{i}$ : $\quad$ stock SI initial affiché dans la période $i=1,2$ avant la passation d'une commande auprès du fournisseur 


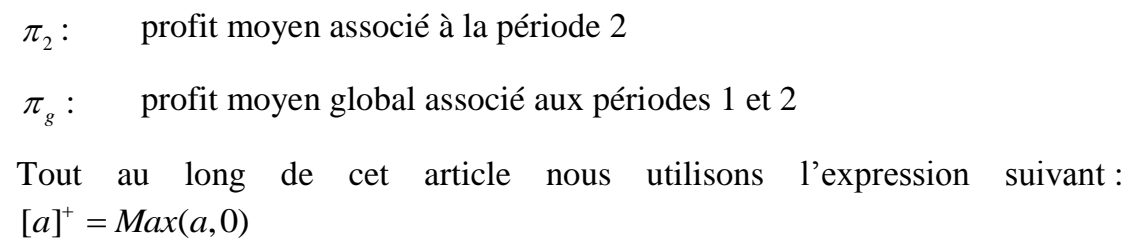

En fonction de la séquence des événements décrite précédemment, nous définissons trois coûts unitaires payés par l'e-détaillant dans sa gestion du stock :

$u_{1}$ : coût unitaire de rupture de type 1 payé quand le niveau affiché dans le IS est insuffisant pour satisfaire une demande, i.e., quand une vente est perdue à cause de la non disponibilité du produit dans le IS.

$u_{2}$ : coût unitaire de rupture de type 2 payé quand le niveau du $\mathrm{PH}$ est insuffisant pour honorer un engagement de vente.

$h_{i}$ : coût unitaire de stockage associé à la période $i=1,2$ correspondant au coût de sur-stockage dans la première période, et au coût payé quand un produit reste invendu à la fin de la deuxième période.

\subsection{Méthodologie de résolution}

Afin de résoudre analytiquement le problème en déterminant les niveaux de recomplètement optimaux $Y_{i}^{*}(i=1,2)$, nous utilisons la programmation dynamique avec une récursion arrière (backward recursion). Ceci consiste à calculer dans un premier temps le profit moyen de la période 2 pour un niveau donné du stock IS initial $x_{2}$ et à déduire dans un second temps le profit global pour les deux périodes de vente en appliquant l'espérance mathématique sur toutes les valeurs possibles de $x_{2}$. Le niveau $x_{2}$ est fonction du niveau de re-complètement de la première période $Y_{1}$ et de la demande $D 1$ générée également durant la première période.

\subsection{Dynamique du système et fonction profit global}

\subsubsection{Analyse du problème mono-période}

Afin de résoudre le problème à deux périodes, il est nécessaire d'étudier dans un premier temps la fonction profit générique relative au problème mono-période. Pour ce faire, nous utilisons la fonction coût associée à une période de vente $i$, cf. équation (1), exprimée un niveau de re-complètement IS $Y_{I S i}$, un niveau de recomplètement PH $Y_{P H i}$, une demande $D i$ et un coût de sur-stockage unitaire $h_{i}$ : 
$L_{i}(Y)=E_{D i}\left[u_{1}\left[D i-Y_{I S i}\right]^{+}+u_{2}\left[\operatorname{Min}\left\{Y_{I S i}, D i\right\}-Y_{P H i}\right]^{+}+h_{i}\left[Y_{P H i}-\operatorname{Min}\left\{Y_{I S i}, D i\right\}\right]^{+}\right]$

Il est à noter que les différentes parties de la fonction coût ci-dessus peuvent être interprétées comme suit :

$u_{1}\left[D i-Y_{I S i}\right]^{+}:$représente la pénalité liée aux pertes de ventes résultant d'une demande cumulative des clients supérieure à la quantité affichée dans le IS.

$u_{2}\left[\operatorname{Min}\left\{Y_{I S i}, D i\right\}-Y_{P H i}\right]^{+}$: représente la pénalité payée si une fraction des engagements de vente n'est pas honorée lors de la livraison aux clients finaux.

$h_{i}\left[Y_{P H i}-\operatorname{Min}\left\{Y_{I S i}, D i\right\}\right]^{+}$: représente la pénalité liée à une situation de surstockage

Pour pouvoir développer la fonction $L_{i}(Y)$ nous utilisons les égalités suivantes (2), (3), (4) et (5) :

$$
\begin{aligned}
& \operatorname{Min}(a, b)=a-[a-b]^{+} \\
& {[a]^{+}=a+[-a]^{+}} \\
& \int_{x=-\infty}^{a} x f_{X}(x) d x=\mu_{X} F_{X}(a)-\sigma_{X}^{2} f_{X}(a) \\
& \int_{x=a}^{+\infty} x f_{X}(x) d x=\mu_{X}\left(1-F_{X}(a)\right)+\sigma_{X}^{2} f_{X}(a)
\end{aligned}
$$

Et en appliquant par la suite l'espérance mathématique par rapport à la demande et aux deux erreurs $D i, Y_{I S i}=\gamma_{I S i} Y_{i}, Y_{P H i}=\gamma_{P H i} Y_{i}$ qui suivent des lois normales, la fonction coût moyen peut être exprimée comme suit (6) :

$$
\begin{aligned}
& L_{i}(Y)=u_{1} \int_{\gamma_{I S i}=0}^{+\infty} \int_{D i=\gamma_{I S I} Y}^{+\infty}\left(D i-\gamma_{I S i} Y\right) f_{D i}(D i) f_{I S i}\left(\gamma_{I S i}\right) d D i d \gamma_{I S i} \\
& +h_{i} \int_{\gamma_{I S i}=0}^{+\infty} \int_{D i=-\infty}^{\gamma_{I S i} Y}\left(\gamma_{I S i} Y-D i\right) f_{D i}(D i) f_{I S i}\left(\gamma_{I S i}\right) d D i d \gamma_{I S i} \\
& +u_{2} Y \int_{\gamma_{P H i}=0}^{+\infty} \int_{\gamma_{I S i}=0}^{+\infty}\left(\gamma_{I S i}-\gamma_{P H i}\right) f_{I S i}\left(\gamma_{I S i}\right) f_{P H i}\left(\gamma_{P H i}\right) d \gamma_{I S i} d \gamma_{P H i} \\
& -\left(u_{2}+h_{i}\right)\left(\begin{array}{l}
\int_{\gamma_{P H i}=0}^{+\infty} \int_{\gamma_{I S i}=0}^{\gamma_{P H i}}\left(\gamma_{I S i}-\gamma_{P H i}\right) Y f_{I S i}\left(\gamma_{I S i}\right) f_{P H i}\left(\gamma_{P H i}\right) d \gamma_{I S i} d \gamma_{P H i} \\
+\int_{\gamma_{I S i}=0}^{+\infty} \int_{\gamma_{P H i}=0}^{\gamma_{I S i}} \int_{D i=\gamma_{P H i} Y}^{\gamma_{I S Y} Y}\left(\gamma_{I S i} Y-D i\right) f_{D i}(D i) f_{P H i}\left(\gamma_{P H i}\right) f_{I S i}\left(\gamma_{I S i}\right) d D i d \gamma_{P H i} d \gamma_{I S i} \\
+\int_{\gamma_{I S i}=0}^{+\infty} \int_{\gamma_{P H i}=0}^{\gamma_{I S i}} \int_{D i=-\infty}^{\gamma_{P H i} Y}\left(\gamma_{I S i}-\gamma_{P H i}\right) Y f_{D i}(D i) f_{P H i}\left(\gamma_{P H i}\right) f_{I S i}\left(\gamma_{I S i}\right) d D i d \gamma_{P H i} d \gamma_{I S i}
\end{array}\right)
\end{aligned}
$$




$$
\begin{aligned}
L_{i}(Y) & =u_{1} \mu_{D i}\left(1-F_{I S i}(0)\right) \\
& +Y \int_{\gamma_{I S i}=0}^{+\infty}\left(\left(u_{1}+h_{i}\right) F_{D i}\left(\gamma_{I S i} Y\right)-u_{1}\right) \gamma_{I S 1} f_{I S 1}\left(\gamma_{I S 1}\right) d \gamma_{I S 1} \\
& -\left(u_{1}+h_{i}\right) \int_{\gamma_{I S i}=0}^{+\infty}\left(\mu_{D i} F_{D i}\left(\gamma_{I S i} Y\right)-\sigma_{D i}^{2} f_{D i}\left(\gamma_{I S i} Y\right)\right) f_{I S i}\left(\gamma_{I S i}\right) d \gamma_{I S i} \\
& +u_{2} Y\left(\mu_{I S i}-\mu_{P H i}\right) \\
& -\left(u_{2}+h_{i}\right)\left(\begin{array}{l}
\int_{\gamma_{P H i}=0}^{+\infty} Y\left(\begin{array}{l}
\left(\mu_{I S i}-\gamma_{P H i}\right)\left(F_{I S i}\left(\gamma_{P H i}\right)-F_{I S i}(0)\right) \\
-\sigma_{I S i}^{2}\left(f_{I S i}\left(\gamma_{P H i}\right)-f_{I S i}(0)\right)
\end{array}\right) f_{P H i}\left(\gamma_{P H i}\right) d \gamma_{P H i} \\
\gamma_{I S i}=0 \\
\left.-\int_{\gamma_{I S i}=0}^{+\infty} \int_{\gamma_{P H i}=0}^{\gamma_{I S i}}\left(\begin{array}{l}
\left(\gamma_{D i}^{2} f_{D i}\left(\gamma_{I S i} Y\right)\right) \\
\left(\gamma_{P H i} Y-\mu_{D i}\right) F_{D i}\left(\gamma_{P H i} Y\right) \\
+\sigma_{D i}^{2} f_{D i}\left(\gamma_{P H i} Y\right)
\end{array}\right) f_{P H i}\left(\gamma_{P H i}\right) f_{I S i}\left(\gamma_{I S i}\right) d \gamma_{P H i} d \gamma_{I S i}\left(\gamma_{I S i}\right)-F_{P H i}(0)\right) f_{I S i}\left(\gamma_{I S i}\right) d \gamma_{I S i}
\end{array}\right)
\end{aligned}
$$

La dérivée première de $L_{i}(Y)$ s'écrit comme suit (7) :

$$
\begin{aligned}
\frac{\partial L_{i}(Y)}{\partial Y}= & \int_{\gamma_{I S i}=0}^{+\infty}\left(\left(u_{1}+h_{i}\right) F_{D i}\left(\gamma_{I S i} Y\right)-u_{1}\right) \gamma_{I S i} f_{I S i}\left(\gamma_{I S i}\right) d \gamma_{I S i} \\
& +u_{2}\left(\mu_{I S i}-\mu_{P H i}\right) \\
& -\left(u_{2}+h_{i}\right)\left(\begin{array}{l}
\int_{\gamma_{P H i}=0}^{+\infty}\left(\begin{array}{l}
\left.\left(\mu_{I S i}-\gamma_{P H i}\right)\left(F_{I S i}\left(\gamma_{P H i}\right)-F_{I S i}(0)\right)\right) \\
-\sigma_{I S i}^{2}\left(f_{I S i}\left(\gamma_{P H i}\right)-f_{I S i}(0)\right)
\end{array}\right) f_{P H i}\left(\gamma_{P H i}\right) d \gamma_{P H i} \\
+\int_{I S i}^{+\infty}\left(\gamma_{I S i} F_{D i}\left(\gamma_{I S i} Y\right)\left(F_{P H i}\left(\gamma_{I S i}\right)-F_{P H i}(0)\right) f_{I S i}\left(\gamma_{I S i}\right) d \gamma_{I S i}\right. \\
-\int_{\gamma_{I S i}=0}^{+\infty} \int_{\gamma_{P H i}=0}^{\gamma_{I S i}}\left(\gamma_{P H i} F_{D i}\left(\gamma_{P H i} Y\right)\right) f_{P H i}\left(\gamma_{P H i}\right) f_{I S i}\left(\gamma_{I S i}\right) d \gamma_{P H i} d \gamma_{I S i}
\end{array}\right)
\end{aligned}
$$

La fonction profit générique pour une période de vente peut être déduite (8), pour un stock initial donné $x_{i}$ :

$$
\pi_{i}\left(x_{i}, Y_{i}\right)=u_{1} \mu_{D i}-L_{i}\left(Y_{i}\right)-c\left(\mu_{P H i} Y_{i}-x_{i}\right)
$$

Pour faciliter la lecture des expressions mathématiques de la solution optimale, nous définissons les constantes suivantes : Pour $i \in\{1,2\}$ 


$$
\begin{aligned}
C_{1 i}= & -\left(u_{1}+h_{i}\right) \int_{0}^{+\infty} \gamma_{I S i} f_{I S i}\left(\gamma_{I S i}\right) \gamma_{I S i} \\
& +\left(u_{2}+h_{i}\right) \int_{0}^{+\infty} \int_{0}^{+\infty}\left(\gamma_{I S i}-\gamma_{P H i}\right) f_{P H i}\left(\gamma_{P H i}\right) f_{I S i}\left(\gamma_{I S i}\right) d \gamma_{P H i} d \gamma_{I S i} \\
C_{2 i}= & -u_{1}\left(\mu_{I S i}\left(1-F_{I S i}(0)\right)+\sigma_{I S i}{ }^{2} f_{I S i}(0)\right)+u_{2}\left(\mu_{I S i}-\mu_{P H i}\right) \\
& -\left(u_{2}+h_{i}\right) \int_{0}^{+\infty}\left(\left(\mu_{I S i}-\gamma_{P H i}\right)\left(F_{I S i}\left(\gamma_{P H i}\right)-F_{I S i}(0)\right)-\sigma_{P H i}^{2}\left(f_{I S i}\left(\gamma_{P H i}\right)-f_{I S i}(0)\right) f_{P H i}\left(\gamma_{P H i}\right) d \gamma_{P H i}\right.
\end{aligned}
$$

Remarque 1: Nous faisons l'hypothèse que la quantité facturée par le fournisseur est la quantité existante réellement en stock (cf. eq. 8 ou le coût unitaire d'achat est appliqué au stock $\mathrm{PH}$ ). Notre hypothèse est motivée par le fait que ce cas de figure est appliqué par exemple pour dans une politique de gestion partagée des approvisionnements (VMI: Vendor Management Inventory). Si nous changeons cette hypothèse et que nous supposons que la facturation pour le fournisseur se base sur la quantité affichée dans le quantités IS (i.e. nous changeons le dernier terme dans eq. 8 par $\left.c\left(\mu_{I S i} Y-x\right)\right)$, notre démarche d'optimisation reste applicable. Le seul paramètre qui change si nous considérons cette hypothèse est $C_{2 i}$ qui devient :

$$
\begin{aligned}
C_{2 i}= & -u_{1}\left(\mu_{I S i}\left(1-F_{I S i}(0)\right)+\sigma_{I S i}{ }^{2} f_{I S i}(0)\right) \\
& +\left(u_{2}-c\right)\left(\mu_{S I i}-\mu_{S P i}\right) \\
& -\left(u_{2}+h_{i}\right) \int_{0}^{+\infty}\left(\begin{array}{l}
\left(\mu_{S I i}-\gamma_{S P i}\right)\left(F_{I S i}\left(\gamma_{S P i}\right)-F_{I S i}(0)\right) \\
-\sigma_{S P i}^{2}\left(f_{I S i}\left(\gamma_{P H i}\right)-f_{I S i}(0)\right)
\end{array}\right) f_{P H i}\left(\gamma_{P H i}\right) d \gamma_{P H i}
\end{aligned}
$$

\subsubsection{Analyse du problème à deux périodes de vente}

Comme mentionné précédemment, nous utilisons la récursion arrière pour résoudre le cas à deux périodes de vente. Pour ce faire, nous commençons par établir les conditions d'optimalité de la période 2 pour un niveau de stock IS initial $x_{2}$ donné. L'optimisation de la période 2 correspond tout simplement au cas monopériode pour un niveau $x_{2}$ donné. En utilisant et en adaptant nos résultats monopériode démontrés dans la section précédente, le niveau de re-complètement optimal pour la période $2, Y_{2}^{*}$ doit satisfaire l'équation (9) qui correspond à l'annulation de la dérivée première de la fonction profit moyen : 


$$
\left.\int_{0}^{+\infty} \int_{0}^{\gamma_{I S 2}}\left[\begin{array}{c}
\left(u_{2}+h_{2}\right) \gamma_{I S 2} F_{D 2}\left(\gamma_{I S 2} Y_{2}^{*}\right) \\
-\left(u_{2}+h_{2}\right) \gamma_{P H 2} F_{D 2}\left(\gamma_{P H 2} Y_{2}^{*}\right) \\
-\left(u_{1}+h_{2}\right) \frac{\gamma_{I S 2} F_{D 2}\left(\gamma_{I S 2} Y_{2}^{*}\right)}{F_{P H 2}\left(\gamma_{I S 2}\right)-F_{P H 2}(0)}
\end{array}\right) f_{P H 2}\left(\gamma_{P H 2}\right)\right) f_{I S 2}\left(\gamma_{I S 2}\right) d \gamma_{P H 2} d \gamma_{I S 2}=C_{22}+c \mu_{P H 2}
$$

Ainsi, pour un stock initial en début de la période 2 égale à $x_{2}$, la quantité que doit commander l'e-détaillant auprès de son fournisseur pour cette période, est égale à $Q_{2}^{*}=\left\{\begin{array}{c}Y_{2}^{*}-x_{2} \text { si } x_{2} \leq Y_{2}^{*} \\ 0 \text { sinon }\end{array}\right.$

Le profit moyen de la période 2 peut également s'écrire comme (10):

$$
\pi_{2}\left(x_{2}, Y_{2}^{*}\right)=\left\{\begin{array}{c}
u_{1} \mu_{D 2}-L_{2}\left(Y_{2}^{*}\right)-c\left(\mu_{P H 2} Y_{2}^{*}-x_{2}\right) \text { si } 0<x_{2} \leq Y_{2}^{*} \\
u_{1} \mu_{D 2}-L_{2}\left(Y_{2}^{*}\right)-c\left(\mu_{P H 2} Y_{2}^{*}\right) \text { si } x_{2}=0 \\
u_{1} \mu_{D 2}-L_{2}\left(x_{2}\right) \text { si } Y_{2}^{*} \leq x_{2}
\end{array}\right.
$$

En utilisant cette dernière expression, le profit moyen global relatif aux deux périodes de vente, après intégration du profit moyen de la période 1 et en appliquant l'espérance mathématique par rapport à $x_{2}$ sur le profit de la période 2, peut se formuler comme suit (11) :

$$
\pi_{g}\left(x_{1}, Y_{1}\right)=\max _{Y_{1} \geq x_{1}}\left\{u_{1} \mu_{D 1}-L_{1}\left(Y_{1}\right)-c\left(\mu_{P H 1} Y_{1}-x_{1}\right)+E_{x_{2}}\left[\pi_{2}^{*}\left(x_{2}, Y_{2}^{*}\right)\right]\right\}
$$

La dynamique du système entre les deux périodes de vente s'exprime par la relation entre $x_{2}$ et la demande de la période 1 :

Le stock disponible au début de période $2, x_{2}$, affiché dans le SI et qui est utilisé pour re-compléter le stock dépend du niveau de re-complètement, des erreurs SI et de la demande, tous relatifs à période 1 :

$$
x_{2}=\left\{\begin{array}{c}
\gamma_{I S 1} Y_{1}-D 1 \text { si } D 1 \leq \gamma_{I S 1} Y_{1} \\
0 \text { si } \gamma_{I S 1} Y_{1} \leq D 1
\end{array}\right.
$$

En appliquant cette dynamique sur l'expression de $\pi_{g}\left(x_{1}, Y_{1}\right)$, le profit moyen des deux périodes de vente peut s'exprimer comme suit (12) : 


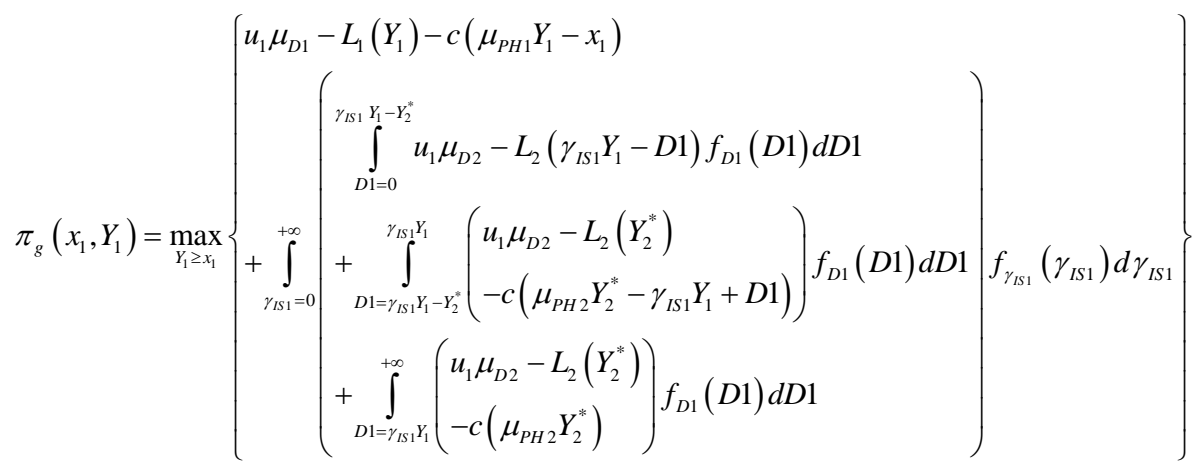

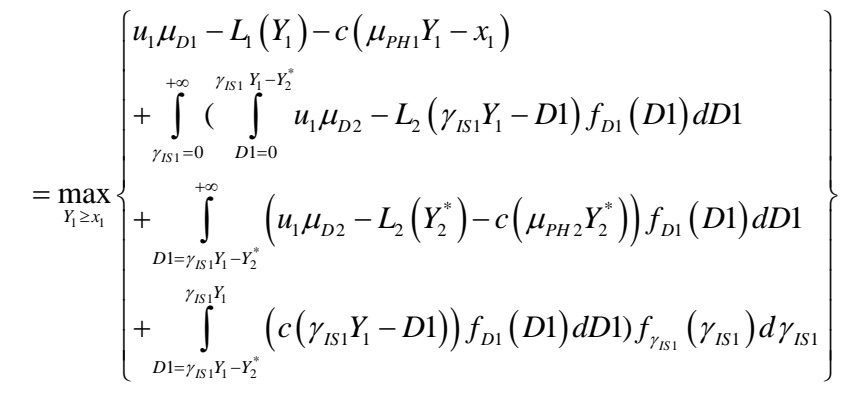

En remplaçant $L_{1}\left(Y_{1}\right), L_{2}\left(\gamma_{I S 1} Y_{1}-D 1\right)$ et $L_{2}\left(Y_{2}^{*}\right)$ par leurs expressions, le profit moyen global peut s'exprimer sous la forme de l'équation (13): 


$$
\begin{aligned}
& \pi_{g}\left(Y_{1}\right)=u_{1}\left(\mu_{D 1}-\mu_{D 1}\left(1-F_{I S 1}(0)\right)\right)-c\left(\mu_{P H 1} Y_{1}-x_{1}\right) \\
& -Y_{1} \int_{\gamma_{S 1}=0}^{+\infty}\left(\left(u_{1}+h_{1}\right) F_{D 1}\left(\gamma_{I S 1} Y_{1}\right)-u_{1}\right) \gamma_{I S 1} f_{I S 1}\left(\gamma_{I S 1}\right) d \gamma_{I S 1} \\
& +\left(u_{1}+h_{1}\right) \int_{\gamma_{S 1}=0}^{+\infty}\left(\mu_{D 1} F_{D 1}\left(\gamma_{I S 1} Y_{1}\right)-\sigma_{D 1}^{2} f_{D 1}\left(\gamma_{I S 1} Y_{1}\right)\right) f_{I S 1}\left(\gamma_{I S 1}\right) d \gamma_{I S 1}-u_{2} Y_{1}\left(\mu_{I S 1}-\mu_{P H 1}\right) \\
& +\left(u_{2}+h_{1}\right)\left(\begin{array}{l}
\int_{\gamma_{P H 1}=0}^{+\infty} Y_{1}\left(\begin{array}{l}
\left(\mu_{I S 1}-\gamma_{P H 1}\right)\left(F_{I S 1}\left(\gamma_{P H 1}\right)-F_{I S 1}(0)\right) \\
-\sigma_{I S 1}^{2}\left(f_{I S 1}\left(\gamma_{P H 1}\right)-f_{I S 1}(0)\right)
\end{array}\right) f_{P H 1}\left(\gamma_{P H 1}\right) d \gamma_{P H 1} \\
+\int_{\gamma_{S L 1}=0}^{+\infty}\left(\begin{array}{l}
\left(\gamma_{I S 1} Y_{1}-\mu_{D D}\right) F_{D 1}\left(\gamma_{I S 1} Y_{1}\right) \\
\left.+\sigma_{D 1}^{2} f_{D 1}\left(\gamma_{I S 1} Y_{1}\right)\right)
\end{array}\right)\left(F_{P H 1}\left(\gamma_{I S 1}\right)-F_{P H 1}(0)\right) f_{I S 1}\left(\gamma_{I S 1}\right) d \gamma_{I S 1} \\
-\int_{\gamma_{S 1}=0}^{+\infty} \int_{\gamma_{P H 1}=0}^{\gamma_{S I 1}=0}\left(\begin{array}{l}
\left(\gamma_{P H 1} Y_{1}-\mu_{D 1}\right) F_{D 1}\left(\gamma_{P H 1} Y_{1}\right) \\
+\sigma_{D 1}^{2} f_{D 1}\left(\gamma_{P H 1} Y_{1}\right)
\end{array}\right) f_{P H 1}\left(\gamma_{P H 1}\right) f_{I S 1}\left(\gamma_{I S 1}\right) d \gamma_{P H 1} d \gamma_{I S 1}
\end{array}\right)
\end{aligned}
$$

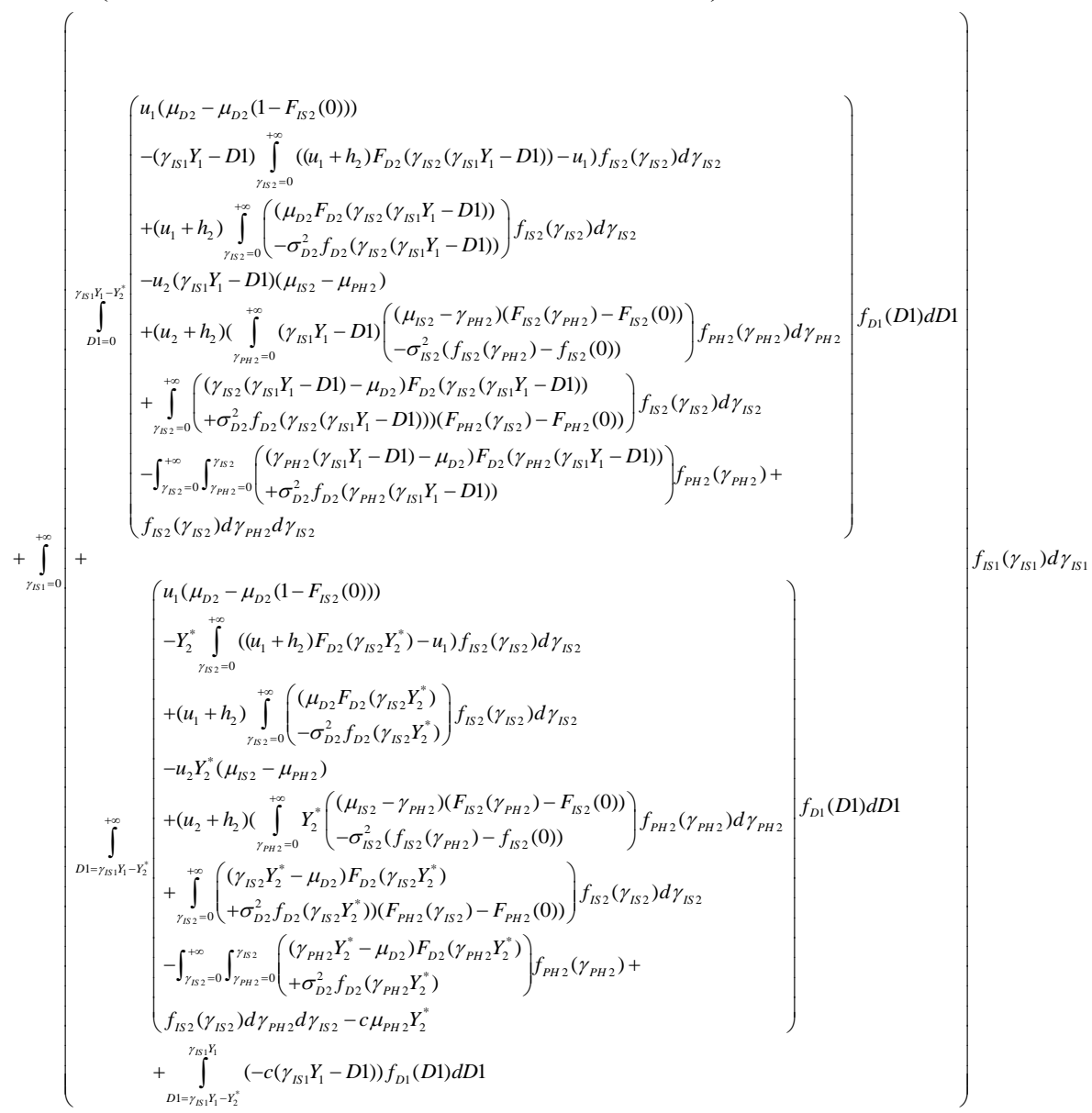




\subsection{Optimisation et conditions d'existence des solutions d'optimalité}

\subsubsection{Conditions d'optimalité pour $Y_{2}^{*}$}

Le but de cette section est de démontrer les conditions d'optimalité des quantités de re-complément pour chaque période de vente. Nous présentons des conditions qui assurent la concavité de la fonction profit moyen. Nous illustrons également le comportement de la fonction profit si la concavité n'est pas assurée et le moyen d'en déduire la solution optimale.

L'optimisation de la période 2 correspond tout simplement au cas mono-période pour un niveau $x_{2}$ donné. En utilisant et en adaptant les résultats mono-période démontrés par Khader et al. (2012), le niveau de re-complètement optimal pour la période $2 Y_{2}^{*}$, s'il existe, doit annuler la dérivée première de la fonction profit moyen qui s'écrit comme suit (14) :

$\frac{\partial \pi_{2}\left(Y_{2}\right)}{\partial Y_{2}}=\int_{0}^{+\infty} \int_{0} \int_{0}\left[\left(\begin{array}{c}\left(u_{2}+h_{2}\right) \gamma_{I S 2} F_{D 2}\left(\gamma_{I S 2} Y_{2}\right) \\ -\left(u_{2}+h_{2}\right) \gamma_{P H 2} F_{D 2}\left(\gamma_{P H 2} Y_{2}\right) \\ \left.-\left(u_{1}+h_{2}\right) \frac{\gamma_{I S 2} F_{D 2}\left(\gamma_{I S} Y_{2}\right)}{F_{P H 2}\left(\gamma_{I S 2}\right)-F_{P H 2}(0)}\right)\end{array}\right) f_{P H 2}\left(\gamma_{P H 2}\right)\right] f_{I S 2}\left(\gamma_{I S 2}\right) d \gamma_{P H 2} d \gamma_{I S 2}-C_{22}-c \mu_{P H 2}$

Afin d'étudier l'existence de la solution optimale $Y_{2}^{*}$, nous analysons le comportement de la dérivée seconde du profit moyen, $\frac{\partial^{2} \pi_{2}\left(Y_{2}\right)}{\partial Y_{2}^{2}}$, qui s'écrit comme suit (15) :

$\frac{\partial^{2} \pi_{2}\left(Y_{2}\right)}{\partial Y_{2}^{2}}=\int_{0}^{+\infty} \int_{0}^{+\infty} \gamma_{S 2}\left[\left(\begin{array}{c}\left(u_{2}+h_{2}\right) \gamma_{I S 2} F_{D 2}\left(\gamma_{I S 2} Y_{2}\right) \\ -\left(u_{2}+h_{2}\right) \gamma_{P H 2} F_{D 2}\left(\gamma_{P H 2} Y_{2}\right) \\ \left.-\left(u_{1}+h_{2}\right) \frac{\gamma_{I S 2} F_{D 2}\left(\gamma_{I S 2} Y_{2}\right)}{F_{P H 2}\left(\gamma_{I S 2}\right)-F_{P H 2}(0)}\right)\end{array}\right) f_{P H 2}\left(\gamma_{P H 2}\right)\right] f_{I S 2}\left(\gamma_{I S 2}\right) d \gamma_{P H 2} d \gamma_{I S 2}$

L'analyse analytique de $\frac{\partial^{2} \pi_{2}\left(Y_{2}\right)}{\partial Y_{2}^{2}}$ montre l'existence d'une hypothèse $\mathrm{H} 1$ permettant de vérifier analytiquement la concavité de la fonction profit moyen. Si la condition H1 n'est pas vérifiée, une analyse numérique approfondie nous montre 
que la dérivée seconde $\frac{\partial^{2} \pi_{2}\left(Y_{2}\right)}{\partial Y_{2}^{2}}$ s'annule uniquement une fois, ce qui nous permet de déduire les conditions d'optimalité de la fonction profit moyen.

$$
\text { H1 : } \mu_{I S 2}+3 \sigma_{I S 2} \leq F_{P H 2}{ }^{-1}\left(\frac{u_{1}+h_{2}}{u_{2}+h_{2}}+F_{P H 2}(0)\right)
$$

\section{$1^{\text {er }}$ cas : Si l'hypothèse H1est verifiée}

Nous supposons que $f_{I S 2}\left(\gamma_{I S 2}\right)=0 s i\left\{\begin{array}{l}\gamma_{I S 2} \leq \mu_{I S 2}-3 \sigma_{I S 2} \\ \mu_{I S 2}+3 \sigma_{I S 2} \leq \gamma_{I S 2}\end{array}\right.$ et donc $\frac{\partial^{2} \pi_{2}\left(Y_{2}\right)}{\partial Y_{2}^{2}}$ peut s'écrire comme suit (16) :

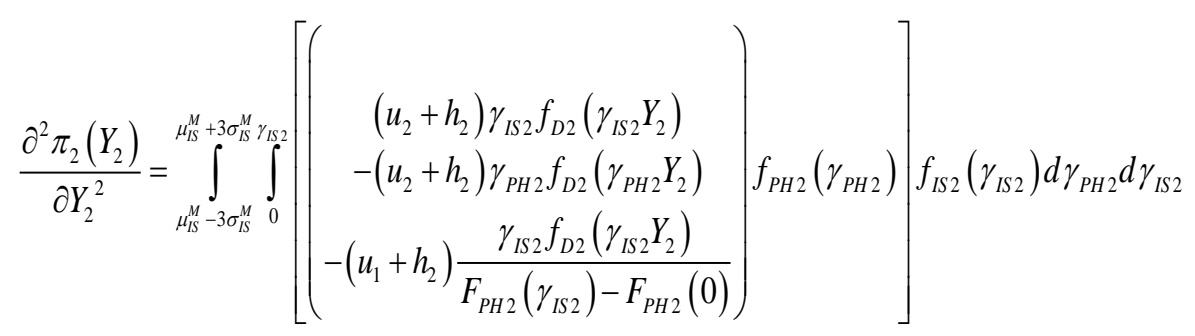

Avec l'hypothèse $\mathrm{H} 1$ nous pouvons conclure que la dérivée seconde $\frac{\partial^{2} \pi_{2}}{\partial Y_{2}^{2}}\left(Y_{2}\right)$ est négative et donc la fonction $\pi_{2}\left(Y_{2}\right)$ est concave.

En définissant $x$ comme étant le ratio entre coût de rupture de type 2 et de type 1 et $y$ le ratio entre coût de stockage et coût de rupture de type 1, i.e. $x=\frac{u_{2}}{u_{1}}$ et $y=\frac{h_{2}}{u_{1}}$, l'hypothèse H1 s'écrit comme suit :

$\mathrm{H} 1: \mu_{I S 2}+3 \sigma_{I S 2} \leq F_{P H 2}{ }^{-1}\left(\frac{1+y}{x+y}+F_{P H 2}(0)\right)$

Les figures 2 et 3 illustrent l'applicabilité de l'hypothèse $\mathrm{H} 1$ c'est-à-dire les valeurs permises par $\sigma_{I S 2}$ en fonction de $x$ et $y$ pour $\mu_{I S 2}=0.8$ et $\mu_{I S 2}=1$ tout en fixant $\mu_{P H 2}=1$ et $\sigma_{P H 2}=0.05$. 
16 JESA. Volume $\mathrm{x}-\mathrm{n}^{\circ} \mathrm{x} / 201 \mathrm{x}$

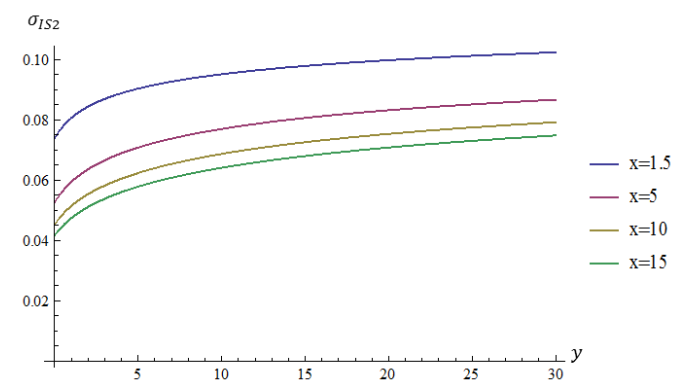

Figure 2. $\sigma_{I S 2}$ en fonction de $x$ et $y$ pour $\mu_{I S 2}=0.8$

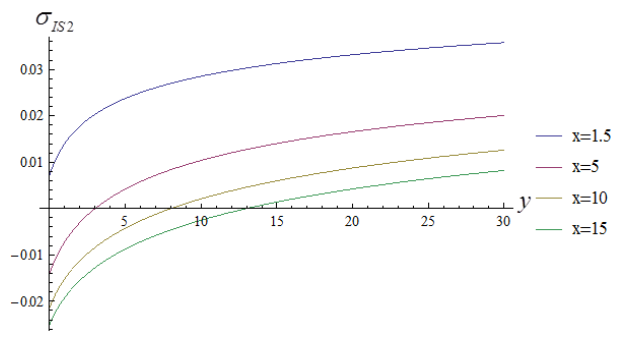

Figure 3. $\sigma_{I S 2}$ en fonction de $x$ et $y$ pour $\mu_{I S 2}=1$

Il est à noter que l'hypothèse $\mathrm{H} 1$ est bel et bien vérifiée pour une variabilité faible de l'erreur IS.

\section{$2^{\text {ème }}$ cas : Si l'hypothèse $H 1$ n'est pas vérifiée}

Pour toute autre combinaison des valeurs d'entrée du problème n'assurant pas l'hypothèse $\mathrm{H} 1$, la fonction profit moyen n'est pas concave. Nous avons réalisé une analyse numérique approfondie avec les valeurs usuelles des coûts et des paramètres des erreurs qui a démontré que la dérivée seconde de la fonction profit, $\frac{\partial^{2} \pi_{2}}{\partial Y_{2}{ }^{2}}\left(Y_{2}\right)$, s'annule au plus une fois. Le Tableau 1 présente à titre d'exemple les intervalles des valeurs prises par les paramètres d'entrée que nous avons testés dans notre analyse numérique. 
Tableau 1. Valeurs prises par les paramètres d'entrées

\begin{tabular}{|c|c|c|c|c|c|c|}
\hline$h_{2}$ & $u_{1}$ & $u_{2}$ & $\mu_{I S 2}$ & $\mu_{P H 2}$ & $\sigma_{I S 2}$ & $\sigma_{P H 2}$ \\
\hline$[1,10]$ & {$[0.1,20]$} & {$\left[u_{1}, 2 u_{1}\right]$} & {$[0.9,1.7]$} & {$[0.9,1.7]$} & {$[0.02,0.17]$} & {$[0.02,0.17]$} \\
\hline
\end{tabular}

Il est à noter que le tableau précédent illustre des valeurs permises par les paramètres d'entrée en cohérence avec les valeurs numériques prises dans d'autres investigations (Sahin et Dallery, 2009).

Si la dérivée seconde s'annule une seule fois, nous pouvons conclure que la dérivée première $\frac{\partial \pi_{2}\left(Y_{2}\right)}{\partial Y_{2}}$ présente au plus un seul optimum. En ajoutant le fait que $\lim _{Y_{2} \rightarrow+\infty} \frac{\partial \pi_{2}}{\partial Y_{2}}=C_{12}-C_{22}$ et $\lim _{Y 2 \rightarrow 0} \frac{\partial \pi_{2}}{\partial Y_{2}}=-C_{22}$, nous déduisons les conditions d'optimalité de la fonction profit moyen qui se résume dans le Tableau 2 cidessous :

Tableau 2. Conditions d'existence de $Y_{2}^{*}$

\begin{tabular}{|c|c|c|c|}
\hline \multicolumn{2}{|c|}{} & $\begin{array}{c}\text { Nombre de } \\
\text { solution pour } \\
(14)\end{array}$ & Optimalité \\
\hline$\left(C_{12}-C_{22}\right) C_{22} \geq 0$ & $C_{22} \leq 0$ & 1 & Solution optimale \\
\hline$\left(C_{12}-C_{22}\right) C_{22} \geq 0$ & $C_{22} \geq 0$ & 1 & Solution non optimale \\
\hline$\left(C_{12}-C_{22}\right) C_{22} \leq 0$ & $C_{22} \leq 0$ & 0 ou 2 & $\begin{array}{c}\text { Si 2 solutions : la plus petite } \\
\text { est optimale }\end{array}$ \\
\hline$\left(C_{12}-C_{22}\right) C_{22} \leq 0$ & $C_{22} \geq 0$ & 0 ou 2 & $\begin{array}{c}\text { Si 2 solutions : la plus grande } \\
\text { est optimale }\end{array}$ \\
\hline Autres combinations & & \multicolumn{2}{|c|}{ Aucune solution } \\
\hline
\end{tabular}

\subsubsection{Conditions d'optimalité pour $Y_{1}^{*}$}

Nous poursuivons dans cette section notre analyse analytique avec la première période de vente et nous étudions les conditions d'optimalité du niveau de recomplètement $Y_{1}^{*}$. 
Si la quantité optimale $Y_{1}^{*}$ existe, son expression doit annuler la dérivée première de la fonction profit moyen associée aux deux périodes de vente, i.e. $Y_{1}^{*}$ doit vérifier $\frac{\partial \pi_{g}}{\partial Y_{1}}\left(Y_{1}^{*}\right)=0$.

Pour calculer $\frac{\partial \pi_{g}}{\partial Y_{1}}\left(Y_{1}\right)$ nous utilisons la formule de Leibniz (17) et l'expression de $\frac{\partial L_{i}(Y)}{\partial Y}(7)$

$$
\frac{\partial \int_{a(y)}^{b(y)} h(x, y) d x}{\partial y}=\int_{a(y)}^{b(y)} \frac{\partial h(x, y)}{\partial y} d x+h(b(y), y) b^{\prime}(y)-h(a(y), y) a^{\prime}(y)
$$

La dérivée première du profit total, $\frac{\partial \pi_{g}}{\partial Y_{1}}\left(Y_{1}\right)$, peut ainsi s'écrire comme suit (18) :

$$
\left.\begin{array}{rl}
\frac{\partial \pi_{g}}{\partial Y_{1}}\left(Y_{1}\right)= & -\frac{d L_{1}\left(Y_{1}\right)}{d Y_{1}}-c \mu_{P H 1} \\
& +\int_{\gamma_{I S 1}=0}^{+\infty} \gamma_{I S 1}\left(\begin{array}{l}
\int_{D 1=0}^{\gamma_{I S 1} Y_{1}-Y_{2}^{*}}-\frac{\partial L_{2}}{\partial Y_{1}}\left(\gamma_{I S 1} Y_{1}-D 1\right) f_{D 1}(D 1) d D 1 \\
+c\left(F_{D 1}\left(\gamma_{I S 1} Y_{1}\right)-F_{D 1}\left(\gamma_{I S 1} Y_{1}-Y_{2}^{*}\right)\right) \\
+c\left(\mu_{P H 2}-1\right) Y_{2}^{*} f_{D 1}\left(\gamma_{I S 1} Y_{1}-Y_{2}^{*}\right)
\end{array}\right) f_{\gamma_{I S 1}\left(\gamma_{I S 1}\right) d \gamma_{I S 1}}
\end{array}\right)
$$




$$
\begin{aligned}
& \frac{\partial \pi_{g}}{\partial Y_{1}}\left(Y_{1}\right)=-\left(u_{1}+h_{1}\right) \int_{\gamma_{I S 1}=0}^{+\infty} F_{D 1}\left(\gamma_{I S 1} Y_{1}\right) \gamma_{I S 1} f_{I S 1}\left(\gamma_{I S 1}\right) d \gamma_{I S 1}-C_{21} \\
& +\left(u_{2}+h_{1}\right)\left(\begin{array}{l}
\int_{\gamma_{I S 1}=0}^{+\infty}\left(\gamma_{I S 1} F_{D 1}\left(\gamma_{I S 1} Y_{1}\right)\right)\left(F_{P H 1}\left(\gamma_{I S 1}\right)-F_{P H 1}(0)\right) f_{I S 1}\left(\gamma_{I S 1}\right) d \gamma_{I S 1} \\
-\int_{\gamma_{I S 1}=0}^{+\infty} \int_{\gamma_{P H 1}=0}^{\gamma_{S I}}\left(\gamma_{P H 1} F_{D 1}\left(\gamma_{P H 1} Y_{1}\right)\right) f_{P H 1}\left(\gamma_{P H 1}\right) f_{I S 1}\left(\gamma_{I S 1}\right) d \gamma_{P H 1} d \gamma_{I S 1}
\end{array}\right)-c \mu_{P H 1}
\end{aligned}
$$

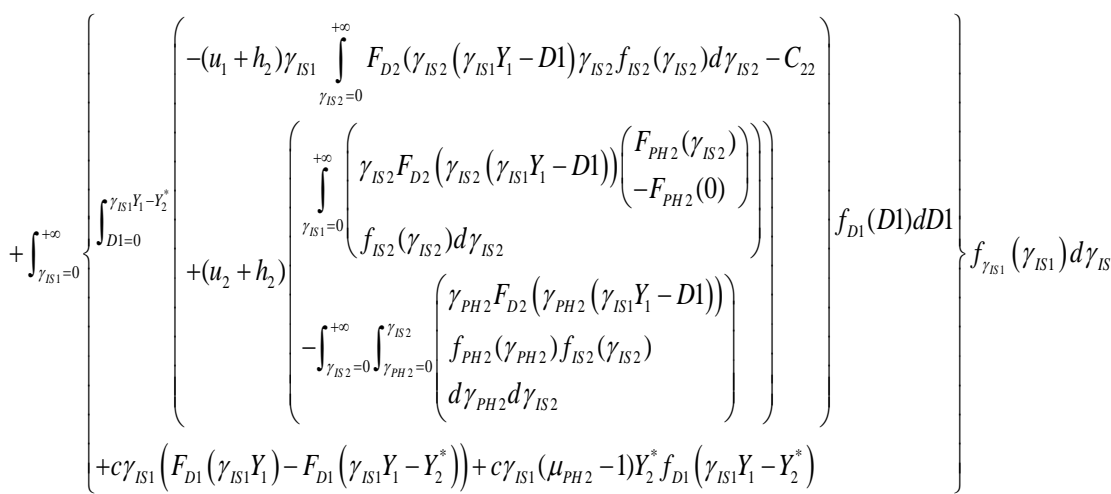

Dans le reste de l'article, pour se focaliser sur l'impact des incertitudes des erreurs et pour simplifier les expressions mathématiques, nous fixons $\mu_{I S 1}=\mu_{I S 2}=\mu_{P H 1}=\mu_{P H 2}=1$.

Comme précédemment, nous analysons les conditions d'optimalité de la fonction profit global des deux périodes en étudiant le comportement de sa dérivée seconde $\frac{\partial^{2} \pi_{g}}{\partial^{2} Y_{1}}\left(Y_{1}\right)$ qui s'écrit comme suit (19) :

$$
\begin{aligned}
& \frac{\partial^{2} \pi_{g}\left(Y_{1}\right)}{\partial^{2} Y_{1}}=-\left(u_{1}+h_{1}-c\right) \int_{\gamma_{S 1}=0}^{+\infty} f_{D 1}\left(\gamma_{I S 1} Y_{1}\right) \gamma_{I S 1}^{2} f_{I S 1}\left(\gamma_{I S 1}\right) d \gamma_{I S 1} \\
& +\left(u_{2}+h_{1}\right)\left(\int_{\gamma_{\zeta 1}=0}^{+\infty}\left(\int_{\gamma_{P H 1}=0}^{\gamma_{S 1}} \gamma_{I S 1}^{2} f_{P 1}^{2}\left(\gamma_{I S 1} f_{D 1}\left(\gamma_{P H 1} Y_{1}\right)\right) f_{P H 1}\left(\gamma_{P H 1}\right) f_{I S 1}\left(\gamma_{I S 1}\right) d \gamma_{P H 1} d \gamma_{I S 1}\right)\right. \\
& \begin{array}{c}
\gamma_{I S 1}^{2}\left(-\left(u_{1}+h_{2}\right) \int_{\gamma_{S 2}=0}^{+\infty} f_{D 2}\left(\gamma_{I S 2}^{2}\left(\gamma_{I S 1} Y_{1}-D 1\right)\right) \gamma_{I S 2} f_{I S 2}\left(\gamma_{I S 2}\right) d \gamma_{I S 2}\right. \\
+\int_{\gamma_{\zeta 1}=0}^{+\infty}\left(\int_{D 1=0}^{\gamma_{I S 1} Y_{1}-\gamma_{2}^{*}}+\left(u_{2}+h_{2}\right)\left(\int_{\gamma_{S 2}=0}^{+\infty} \int_{\gamma_{P H 2}=0}^{\gamma_{S S 2}}\left(\begin{array}{l}
\gamma_{I S 2}^{2} f_{D 2}\left(\gamma_{I S 2}\left(\gamma_{I S 1} Y_{1}-D 1\right)\right. \\
-\gamma_{P H 2}^{2} f_{D 2}\left(\gamma_{P H 2}\left(\gamma_{I S 1} Y_{1}-D 1\right)\right)
\end{array}\right) f_{P H 2}\left(\gamma_{P H 2}\right) f_{I S 2}\left(\gamma_{I S 2}\right) d \gamma_{P H 2} d \gamma_{I S 2}\right)\right) f_{D 1}(D 1) d D 1
\end{array} \\
& \left.-c \gamma_{I S 1}^{2} f_{D 1}\left(\gamma_{I S 1} Y_{1}-Y_{2}^{*}\right)\right) f_{I S 1}\left(\gamma_{I S 1}\right) d \gamma_{I S 1}
\end{aligned}
$$


Comme dans la section précédente, nous définissons une nouvelle hypothèse $\mathrm{H} 2$ qui porte cette fois sur les paramètres d'entrée associés à la première période de vente :

H2: $\mu_{I S 1}+3 \sigma_{I S 1} \leq F_{P H 1}{ }^{-1}\left(\frac{u_{1}+h_{1}-c}{u_{2}+h_{1}}+F_{P H 1}(0)\right)$

Et en posant $w=\frac{u_{2}}{u_{1}}$ et $z=\frac{h_{1}}{u_{1}}$ nous pouvons illustrer $\mathrm{H} 2$ avec les figures 4 et 5 qui représentent les valeurs permises par de $\sigma_{I S 1}$ en fonction de $w$ et $z$ pour $\mu_{I S 1}=0.8$ et $\mu_{I S 1}=1$ avec $\mu_{P H 1}=1$ et $\sigma_{P H 1}=0.05$ et $c=2$.

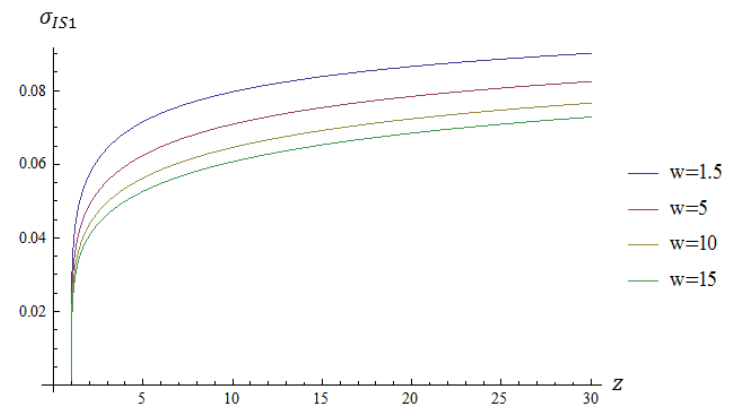

Figure 4. $\sigma_{I S 1}$ en fonction de $w$ et $z$ pour $\mu_{I S 1}=0.8$

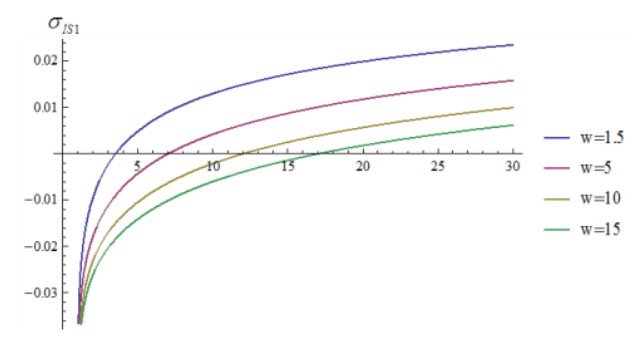

Figure 5. $\sigma_{I S 1}$ en fonction de $w$ et $z$ pour $\mu_{I S 1}=1$

Comme pour $\mathrm{H} 1$, il est à noter que $\mathrm{H} 2$ est verifiée pour des faibles variabilités de l'erreur IS de la première période de vente.

Afin de pouvoir l'étudier, nous décomposons $\frac{\partial^{2} \pi_{g}}{\partial^{2} Y_{1}}\left(Y_{1}\right)$ comme suit : 


$$
\frac{\partial^{2} \pi_{g}}{\partial^{2} Y_{1}}\left(Y_{1}\right)=P T 1\left(Y_{1}\right)-P T 2\left(Y_{1}\right)
$$

Où $P T 1\left(Y_{1}\right)$ (respectivement $\left.P T 2\left(Y_{1}\right)\right)$ a pour expression (20) (respectivement (21)) :

$$
\begin{aligned}
& P T 1\left(Y_{1}\right)=\int_{\gamma_{I S 1}=0}^{+\infty}\left(\begin{array}{l}
-\left(u_{1}+h_{1}-c\right) \\
+\left(u_{2}+h_{1}\right)\left(F_{P H 1}\left(\gamma_{I S 1}\right)-F_{P H 1}(0)\right)
\end{array}\right) f_{D 1}\left(\gamma_{I S 1} Y_{1}\right) f_{I S 1}\left(\gamma_{I S 1}\right) d \gamma_{I S 1}
\end{aligned}
$$

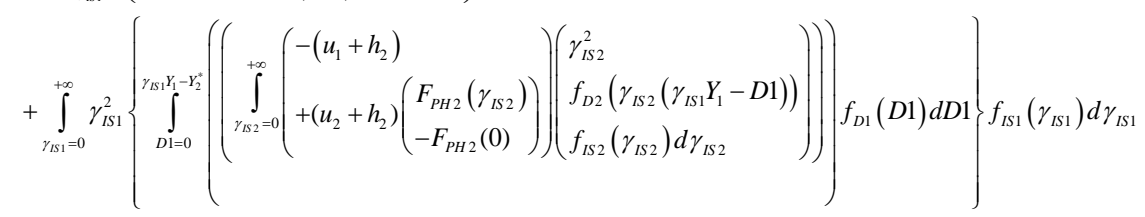

$$
\begin{aligned}
& \operatorname{PT} 2\left(Y_{1}\right)= \\
& \begin{array}{l}
\left(u_{2}+h_{1}\right) \int_{0}^{+\infty} \int_{\gamma_{P H 1}=0}^{\gamma_{I S 1}}\left(\gamma_{P H 1}^{2} f_{D 1}\left(\gamma_{P H 1} Y_{1}\right)\right) f_{P H 1}\left(\gamma_{P H 1}\right) f_{I S 1}\left(\gamma_{I S 1}\right) d \gamma_{P H 1} d \gamma_{I S 1} \\
\left.+\int_{\gamma_{I S 1}=0}^{+\infty} f_{P H 2}\left(\gamma_{P H 2}\right) f_{I S 1}\left(\gamma_{I S 2}\right) h_{D 1}\right) \gamma_{I S 2}^{2} \int_{D 1=0}^{\gamma_{I S 1} Y_{1}-Y_{2}^{*}} \int_{0}^{+\infty} \int_{\gamma_{P H}=0}^{\gamma_{I S 2}} \gamma_{P H 2}^{2} f_{D 2}\left(\gamma_{P H 2}\left(\gamma_{I S 1} Y_{1}-D 1\right) d \gamma_{P H 2} d \gamma_{I S 2} d D 1\right. \\
\left.-c \gamma_{I S 2}^{2} f_{D 1}\left(\gamma_{I S 1} Y_{1}-Y_{2}^{*}\right)\right) f_{I S 1}\left(\gamma_{I S 1}\right) d \gamma_{I S 1}
\end{array}
\end{aligned}
$$

Si les hypothèses $\mathrm{H} 1$ et $\mathrm{H} 2$ sont vérifiées les conditions d'optimalité des niveaux de re-complètement peuvent être déduites comme suit :

$P T 1\left(Y_{1}\right)$ est une fonction négative et $P T 2\left(Y_{1}\right)$ est une fonction positive donc $\frac{\partial^{2} \pi_{g}}{\partial^{2} Y_{1}}\left(Y_{1}\right)$ est négative. D'où nous pouvons conclure que $\frac{\partial \pi_{g}}{\partial Y_{1}}\left(Y_{1}\right)$ est une fonction décroissante et nous posons $C_{\infty}=\lim _{Y_{1} \rightarrow+\infty} \frac{\partial \pi_{g}\left(x_{1}, Y_{1}\right)}{\partial Y_{1}}=C_{11}-C_{21}+C_{12}-C_{22}$ et $\lim _{Y 1 \rightarrow 0} \frac{\partial \pi_{g}\left(x_{1}, Y_{1}\right)}{\partial Y_{1}}=-C_{21}$.

L'allure de $\frac{\partial^{2} \pi_{g}}{\partial^{2} Y_{1}}\left(Y_{1}\right)$ est représentée dans la figure 6. 


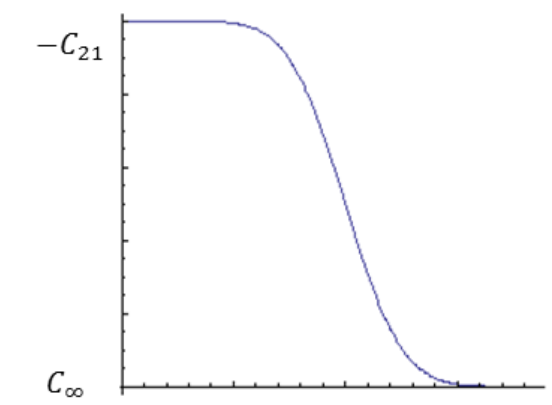

Figure 6.Variations de $\frac{\partial^{2} \pi_{g}}{\partial^{2} Y_{1}}\left(Y_{1}\right)$ en fonction de $Y_{1}$

Le tableau 3 résume les conditions d'existence d'une solution optimale pour le problème d'incertitude sur les stocks sur deux périodes et avec une modélisation multiplicative des erreurs.

Tableau 3. Conditions d'existence $Y_{1}^{*}$

\begin{tabular}{|c|c|c|c|}
\hline \multicolumn{2}{|c|}{} & $\begin{array}{c}\text { Nombre de solution } \\
\text { de (18) }\end{array}$ & Optimalité \\
\hline$-C_{21} C_{\infty} \leq 0$ & $-C_{21} \geq 0$ & 1 & Optimal \\
\hline$-C_{21} C_{\infty} \leq 0$ & $-C_{21} \leq 0$ & 1 & Pas optimal \\
\hline$-C_{21} C_{\infty} \geq 0$ & $-C_{21} \geq 0$ & 0 ou 2 & $\begin{array}{c}\text { Si 2 solutions : la plus } \\
\text { petite est optimale }\end{array}$ \\
\hline$-C_{21} C_{\infty} \geq 0$ & $-C_{21} \leq 0$ & 0 ou 2 & $\begin{array}{c}\text { Si 2 solutions : la plus } \\
\text { grande est optimale }\end{array}$ \\
\hline $\begin{array}{c}\text { Autre } \\
\text { Combinaisons }\end{array}$ & \multicolumn{3}{|c}{ Pas de solution } \\
\hline
\end{tabular}

Dans la résolution mathématique, nous remarquons que la dérivée de la fonction profit moyen global peut avoir 2, 1 ou 0 solution. Et, si elle admet une solution, cette dernière peut être un minimum et non un maximum.

Si les hypothèses $\mathrm{H} 1$ et $\mathrm{H} 2$ ne sont pas vérifiées, il n'existe pas de règles générales décrivant les conditions optimales du niveau de re-complètement à cause du nombre important de paramètres dont dépend la fonction profit global moyen, l'étude de son comportement devient très difficile à cause du caractère multiplicatif 
des perturbations, et donc afin de trouver une solution, si elle existe, il faudra tracer la dérivée afin de voir les points où cette dernière s'annule.

\section{Application numérique}

Nous présentons dans cette section, une analyse numérique afin de répondre à des interrogations managériales sur l'impact des erreurs IS et PH sur la stratégie optimale de chaque période de vente.

Nous considérons un e-détaillant ayant des produits à forte marge $\left(u_{1}\right.$ et $u_{2}$ sont supérieurs à $h_{1}$ et $\left.h_{2}\right)$ et des produits à faible marge $\left(u_{1}\right.$ et $u_{2}$ sont inférieurs à $h_{1}$ et $\left.h_{2}\right)$. Cette distinction est faite afin de permettre une analyse complète. Le tableau 4 présente les valeurs relatives aux coûts unitaires considérés dans notre étude numérique ainsi que les paramètres de la demande pour chaque période de vente. Ce choix de valeurs d'entrée du problème a été fait en se basant sur des valeurs numériques prises dans les travaux de Atali et al. (2009), Kök et Shang (2007) et Lee et Özer (2007).

Tableau 4. Valeurs des paramètres de l'étude numérique

\begin{tabular}{|c|c|c|}
\hline Coût unitaire & Produit à forte marge & Produit à faible marge \\
\hline$u_{1}$ & 15 & 0.5 \\
\hline$u_{2}$ & 25 & 0.8 \\
\hline$c$ & 2 & 2 \\
\hline$h_{1}$ & 3 & 3 \\
\hline$h_{2}$ & 1 & 1 \\
\hline$\mu_{D 1,2}$ & 20 & 4 \\
\hline$\sigma_{D 1,2}$ & 4 & 5 \\
\hline$x_{1}$ & 5 & \\
\hline
\end{tabular}

Notre analyse numérique a montré que les écarts types des perturbations $\sigma_{I S}$ et $\sigma_{P H}$ ont un rôle symétrique dans la solution optimale, c'est pourquoi nous limitons dans la suite notre illustration graphique à l'impact de $\sigma_{I S}$. 
Pour chaque type de produit (forte ou faible marge) nous faisons varier $\sigma_{I S 2}$ pour différentes valeurs de $\sigma_{I S 1}$. Afin de se focaliser sur l'impact des incertitudes, nous fixons $\mu_{I S 1}=\mu_{I S 2}=\mu_{P H 1}=\mu_{P H 2}=1$

Les figures 7 et 8 (respectivement figures 9 et 10) présentent la variation de $Y_{1}^{*}$ et $Y_{2}^{*}$ en fonction des écarts types des perturbations IS et $\mathrm{PH}$ pour les produits à forte marge (respectivement faible marge).

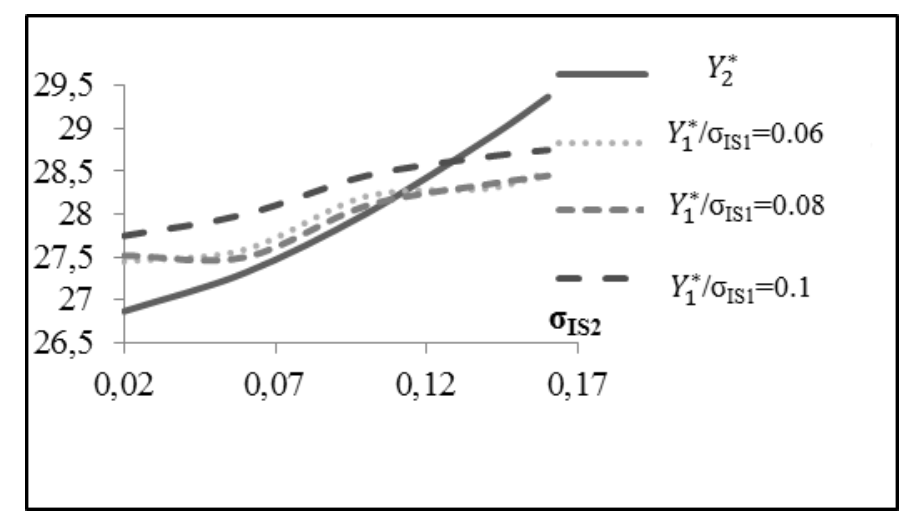

Figure. 7. $Y_{1}^{*}$ et $Y_{2}^{*}$ fonction de $\sigma_{I S 1}$ et $\sigma_{I S 2}$ pour un produit à forte marge

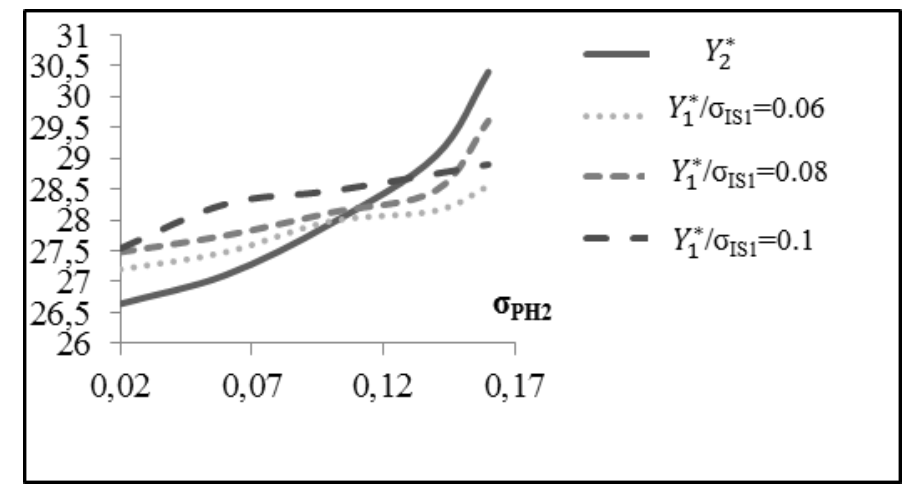

Figure.8. $Y_{1}^{*}$ et $Y_{2}^{*}$ fonction de $\sigma_{P H 1}$ et $\sigma_{P H 2}$ pour un produit à forte marge

$Y_{1}^{*}$ et $Y_{2}^{*}$ se comportent de la même manière face à la variabilité des perturbations IS et $\mathrm{PH}$ : 
- pour les produits à forte marge, $Y_{1}^{*}$ et $Y_{2}^{*}$ augmentent avec la variabilité des perturbations IS et $\mathrm{PH}$ ( $1^{\text {ère }}$ et $2^{\text {ème }}$ période) afin d'éviter la situation de rupture qui est plus pénalisante que la situation de sur-stockage en cas de produit à forte marge.

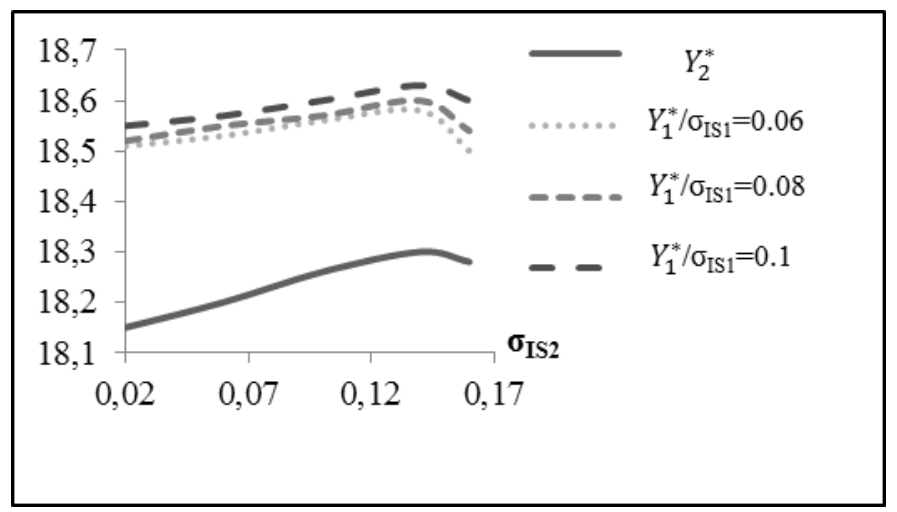

Figure. 9. $Y_{1}^{*}$ et $Y_{2}^{*}$ fonction de $\sigma_{I S 1}$ et $\sigma_{I S 2}$ pour un produit à faible marge

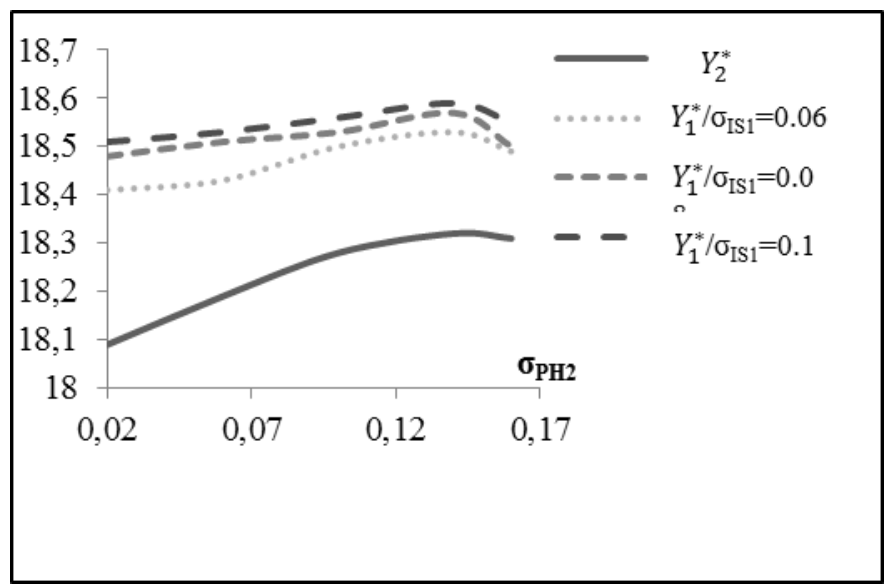

Figure. 10. $Y_{1}^{*}$ et $Y_{2}^{*}$ fonction de $\sigma_{P H 1}$ et $\sigma_{P H 2}$ pour un produit à faible marge

- pour les produits à faible marge, la quantité optimale à avoir en stock en début de chaque période $\left(Y_{1}^{*}\right.$ et $Y_{2}^{*}$ ) augmente dans un premier temps afin d'éviter des situations de rupture résultant des perturbations. La pente de la courbe s'inverse 
ensuite et devient décroissante pour les grandes valeurs de la variabilité des perturbations IS et $\mathrm{PH}$ ( $1^{\text {ère }}$ et $2^{\text {ème }}$ période). Un tel changement de pente pourrait être expliqué par le fait que pour les grandes valeurs de la variabilité, l'augmentation de $Y_{1}^{*}$ et $Y_{2}^{*}$ contribue à l'augmentation de l'imprécision car les perturbations sont multiplicatives. L'augmentation des stocks physiques et par conséquent l'augmentation de la probabilité d'avoir une situation de sur-stockage est plus importante. Il est à rappeler que dans le cas des produits à faible marge, la situation de sur-stockage est plus pénalisante en terme économique que la situation de rupture ;

Dans tous les cas, nous remarquons que le profit moyen optimum est décroissant avec la variabilité des perturbations SI ( $1^{\text {ère }}$ et $2^{\text {ème }}$ période) car plus l'incertitude est grande, plus le profit est négativement impacté par les risques de ruptures et surstockage causés par les perturbations.

Une comparaison entre $Y_{1}^{*}$ et $Y_{2}^{*}$ est réalisée afin de déduire comment le risque des erreurs doit être partagé entre les deux périodes de vente. Ceci nous conduit aux remarques suivantes :

- pour les produits à faible marge nous remarquons que $Y_{1}^{*}>Y_{2}^{*}$ : ceci peut s'expliquer par le fait qu'il est moins risqué de commander beaucoup la première période pour faire face aux perturbations et de reporter les quantités invendues sur la deuxième période. L'augmentation de $Y_{1}^{*}$ entraine mécaniquement l'augmentation de $x_{2}$ et donc la quantité à commander pour la période 2 est moins importante afin d'éviter la situation de sur-stockage ;

- pour les produits à forte marge la situation est différente :

- pour les petites valeurs de $\sigma_{S I 2}$ et $\sigma_{P H 2}$ nous avons peu d'incertitude sur $Y_{2}^{*}$ mais l'incertitude sur $Y_{1}^{*}$ est importante car $Y_{1}^{*}$ est fonction à la fois de $\sigma_{I S 1}$ et de $\sigma_{I S 2}$ (ainsi que de $\sigma_{P H 1}$ et $\sigma_{P H 2}$ ). Constatant que pour les produits à forte marge, les ventes perdues et les engagements de vente non honorés sont plus pénalisants que les situations de sur-stockage, il est intuitif d'expliquer l'augmentation de $Y_{1}^{*}$;

- pour les grandes valeurs de $\sigma_{I S 2}$ et $\sigma_{P H 2}$, la variabilité des perturbations de la période 2 l'emporte sur celle de la première période.

A cause des remarques précédentes, nous concluons cette analyse de comparaison par le fait qu'une solution myopique ne serait pas optimale si nous voulons étendre le problème vers la configuration multi périodes car les niveaux de re-complètement ne sont pas toujours croissants avec les périodes de vente.

\section{Conclusion}

Dans ce papier, nous avons étudié l'impact des perturbations de stock sur la politique de réapprovisionnement dans un contexte e-détaillant en proposant une résolution du problème avec une modélisation multiplicative des erreurs afin 
d'optimiser le profit. Nous avons étendu les résultats existants vers le cas à deux périodes de vente avec une résolution analytique du problème. Les conditions d'optimalité de la stratégie de commande pour les deux périodes de vente ont été démontrées et peuvent ainsi être aisément déployées dans un contexte pratique.

En addition à la contribution théorique, l'étude numérique a permis de répondre à des interrogations managériales concernant le partage des risques des perturbations entre les deux périodes de vente. Ce partage de risque dépend essentiellement de la marge du produit considéré et du comparatif des variabilités des erreurs entre les deux périodes.

Pour les produits à faible marge, la pente des courbes de $Y_{1}^{*}$ et $Y_{2}^{*}$ change suivant la valeur de la variabilité des perturbations. Pour les produits à forte marge, $Y_{1}^{*}$ et $Y_{2}^{*}$ augmentent avec la variabilité des perturbations.

De plus, nous remarquons que le développement analytique a atteint des limites mathématiques avec l'extension de une à deux périodes de vente. Les quantités optimales n'ont pas un comportement monotone avec la variabilité des perturbations, car suivant le type de produit (forte ou faible marge) et la valeur des variabilités des perturbations de la $1^{\text {ère }}$ et $2^{\text {ème }}$ nous n'avons pas une relation monotone entre $Y_{1}^{*}$ et $Y_{2}^{*}$. Cette non monotonie rendra très difficile voire impossible l'utilisation des résultats mono-période dans le cas multi-périodes. En effet, à cause de ce comportement non monotone des quantités optimales, une solution myopique n'est pas optimale pour le cas à plusieurs périodes. Afin de contourner cette situation de complexité et d'étendre la configuration multiplicative au cas multi-périodes, nous préconisons l'utilisation d'une approche d'optimisation basée sur le taux de service pour de futurs développements.

Remerciements: Nous tenons à remercier la Région Rhône-Alpes, cluster de recherche GOSPI, pour son soutien financier sur ce projet de recherche.

\section{Références}

Atali A., Lee H., Ozer O., (2009). If the inventory manager knew: Value of RFID under imperfect inventory information. Tech. rep., Graduate School of Business, Stanford University. URL available at http://ssrn.com/abstract=1351606.

DeHoratius N., Raman A., (2008). Inventory record inaccuracy: an empirical analysis. Management science, 54, 627-641.

Fleisch E., Tellkamp C., (2005). Inventory inaccuracy and supply chain performance: a simulation study of a retail supply chain. International Journal of Production Economics 95(3): 373-385.

Heese H. (2007). Inventory record inaccuracy, double, marginalization and RFID adoption. Production and Operations Management, 16, 542-553. 
Khader S., Rekik Y., Syntetos A., Campagne J. P., Botta-Genoulaz V., (2012). The inventory inaccuracy issue under a multiplicative error structure. INCOM Conference, Bucharest, Romania, Mai 23-25, 2012.

Kök A. G., Shang H. K., (2007). Replenishment and inspection policies for systems with inventory inaccuracy. Manufacturing and Service Operations Management Science, 27, 537-553.

Lee H. L., Özer O. (2007) Unlocking the Value of RFID. Production and Operations Management, 16(1), 40-64.

Nachtmann H., Waller M., Rieske D.W., (2010). The impact of point of sale data inaccuracy and inventory record data errors. Journal of Business Logistics, 31(1), 149-158.

Raman A., DeHoratius N., Ton Z., (2001) Execution: the missing link in retail operations. California Management Review, 43, 136-152.

Rekik Y. (2011). Inventory inaccuracies in the wholesale supply chain. International Journal of Production Economics, 133, 172-181.

Rekik Y., Sahi E., Dallery Y., (2008). Analysis of the impact of the RFID technology on reducing product misplacement errors at retail stores. International Journal of Production Economics, 112, 264-278.

Sahin E., Dallery Y., (2009). Assessing the impact of inventory inaccuracies with in a newsvendor framework. European Journal of Operational Research, 197, 108-118.

Sandoh H., Shimamoto H., (2001). A theoretical study on optimal inventory taking frequency for retailing. Journal of Retailing and Consumer Services, 8(1), 47-52.

Schrady D. A. (1970). Operational definitions of inventory record accuracy. Naval Research Logistics Quarterly, 17, 133-142.

Ton Z., Raman A., (2010). The effect of product variety and inventory levels on misplaced products at retail stores: a longitudinal study. Production and Operations Management, $19,546-560$.

Xu J., Jiang W., Feng G., Tian J., (2012). Comparing improvement strategies for inventory inaccuracy in a two-echelon supply chain”. European Journal of Operational Research 221(1):213-221. 